\title{
Benefits of genetic engineering for human welfare.
}

\author{
Kulkarni M* \\ Department of Biotechnology, Walchand Centre for Biotechnology, Solapur, India
}

Accepted on August 04, 2020

\begin{abstract}
Editorial
"Genetic Engineering or recombinant DNA technology is a strategy utilizing which the hereditary synthesis of a cell can be modified." Genetic manipulation, genetic modification or genetic alterations, broadly it is categorized as genetic engineering. Today there are so many applications are enhancing particularly in this domain. Genetic Engineering has huge applications in field of Environmental Science, Medicine, Food Industry, Agriculture and so on. Genetic engineering mainly focused on Gene isolation, Gene modification, Gene removal and Gene recombination. With all this the final aim of genetic engineering is to improvise the life of human being [1,2].
\end{abstract}

\section{Application of Genetic Engineering}

\section{Food Industry}

Genetically modified (GE) food supplements have had their DNA changed utilizing genes from different plants or creatures. Researchers take the quality for an ideal attribute in one plant or creature, and they embed that quality into a cell of another plant or creature. Hereditarily changed nourishments are gotten from hereditarily adjusted creatures, or transgenic crops. Hereditary building has brought about various improved characteristics in transgenic plants by hereditary adjustment. Benefits of genetically modified food are as follows: more nutritious food, tastier food, Disease- and drought-resistant plants that require fewer environmental resources (such as water and fertilizer), Increased supply of food with reduced cost and longer shelf life etc. [3].

\section{Medicine}

In medication, genetic engineering has been utilized to massproduce insulin, human development hormones, follistim (for rewarding infertility), human albumin, monoclonal antibodies, antihemophilic variables, immunizations, and numerous different medications. Pharming involves the use of genetic engineering technique to insert genes into host animals or plants resulting in expression of useful pharmaceuticals products. The recombinant proteins produced by pharming acts as drugs for various human diseases. These therapeutic products can be directly injected into the bodies of the patient to treat the disease and deficiency. Vaccine and gene therapy has made a remarkable achievement to overcome consequences of several critical and pandemic diseases. This all medicinal miracles are possible because of genetic engineering.

\section{Application in Environment}

Genetic engineering is by using of microorganisms, plants, animals for the restoration of the environment. Genetic engineering is actively involved in the development of microorganisms and biocatalysts for remediation of contaminated environments, and in development of eco-friendly processes such as developing recombinant strain for bio-fuel production etc. Genetically modified organisms are used in clearing up of oil spills which is a major environmental hazard. Increased level of carbon dioxide is directly linked to global warming and greenhouse effect. So efforts are being made to reduce the atmospheric $\mathrm{CO}_{2}$ concentration. In this context, the enzyme ribulose biphosphate carboxylase (RUBP-case) which is closely linked with $\mathrm{CO}_{2}$ fixation is being designed in a manner which results in increased photosynthetic efficiency. New strains of microalgae like mutants of Anacystis nidulans and Oocystis sp are being developed which can tolerate high concentrations of $\mathrm{CO}_{2}$.

\section{Crop improvement and transgenesis}

Transgenic plants can be designed to produce a variety of useful compounds, like therapeutic products and metabolites. Recently transgenic crops with combined traits like herbicide tolerant and insect resistant has been developed.

\section{Improvement of animals through transgenesis}

Genetic Engineering includes the acquaintance of transgene into animal with improve the attribute of transgenic animals. Transgenic animals in this manner at long last express the attribute of the presented quality. Transgenic animals are additionally made to contemplate the capacity of various qualities to create legitimate treatment of diseases [4].

\section{References}

1. http://epgp.inflibnet.ac.in/epgpdata/uploads/epgp_content/ S000002BI/P001357/M021491/ET/1501755083geneticengi neeringetextpathshaala(corrected.pdf

2. https://medlineplus.gov/ency/article/002432.

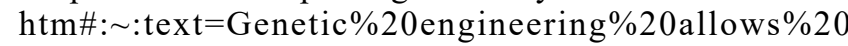
scientists\%20to,plant\%20or\%20animal $\% 20$ into $\% 20$ another.\&text $=$ Another $\% 20$ name $\% 20$ for $\% 20$ this $\% 20$ is,desired $\% 20$ traits $\% 20$ and $\% 20$ breeding $\% 20$ them.

3. https://geneticeducation.co.in/what-is-genetic-engineeringdefinition-types-process-and-application/

4. https://www.biologydiscussion.com/genetic-engineering/ applications-genetic-engineering/top-4-applications-ofgenetic-engineering/37350

\section{*Correspondence to:}

Kulkarni M, Department of Biotechnology, Walchand Centre for Biotchnology, Solapur, India; E-mail: mskulkarni@gmail.com 